\title{
Strain-Promoted Azide-Alkyne Cycloaddition-Based PSMA-Targeting Ligands For Multimodal Intraoperative Tumor Detection of Prostate Cancer
}

Yvonne H.W. Derks ( $\square$ yvonne.derks@radboudumc.nl )

Radboud university medical center https://orcid.org/0000-0002-8512-4103

Mark Rijpkema

Radboud university medical center

Helene I.V. Amatdjais-Groenen

Radboud University: Radboud Universiteit

Cato Loeff

Radboud university medical center

Kim E. de Roode

Radboud university medical center

Annemarie Kip

Radboud university medical center

Peter Laverman

Radboud university medical center

\section{Susanne Lütje}

University Hospital Bonn: Universitatsklinikum Bonn

\section{Sandra Heskamp}

Radboud university medical center

Dennis W.P.M. Löwik

Radboud University: Radboud Universiteit

\section{Research Article}

Keywords: Prostate specific membrane antigen (PSMA), Strain-promoted azide-alkyne cycloaddition (SPAAC), prostate cancer (PCa), multimodal imaging

Posted Date: September 29th, 2021

DOI: https://doi.org/10.21203/rs.3.rs-925760/v1

License: (a) (i) This work is licensed under a Creative Commons Attribution 4.0 International License. Read Full License 
Version of Record: A version of this preprint was published at Bioconjugate Chemistry on December 25th, 2021. See the published version at https://doi.org/10.1021/acs.bioconjchem.1c00537. 


\section{Abstract}

Purpose: Strain-promoted azide-alkyne cycloaddition (SPAAC) is a straightforward and multipurpose conjugation strategy. Use of SPAAC to link different functional elements to prostate specific membrane antigen (PSMA) ligands would facilitate the development of a modular platform for PSMA-targeted imaging and therapy of prostate cancer (PCa). As a first proof-of-concept for the SPAAC chemistry platform we synthesized and characterized four dual-labeled PSMA ligands for intraoperative radiodetection and fluorescence imaging of $\mathrm{PCa}$.

Methods: Ligands were synthesized using solid phase chemistry and contained a chelator for ${ }^{111}$ In or ${ }^{99 m}$ Tc labeling. The fluorophore IRDye800CW was conjugated using SPAAC chemistry or conventional $\mathrm{N}$ hydroxysuccinimide (NHS)-ester coupling. LogD values were measured and PSMA-specificity of these ligands was determined in LS174T-PSMA cells. Tumor targeting was evaluated in BALB/c nude mice with subcutaneous LS174T-PSMA and LS174T wildtype tumors using $\mu$ SPECT/CT imaging, fluorescence imaging, and biodistribution studies.

Results: SPAAC chemistry increased lipophilicity of the ligands (range LogD: -2.4 to -4.4). In vivo, SPAAC chemistry ligands showed high and specific accumulation in s.c. LS174T-PSMA tumors up to 24 hours after injection, enabling clear visualization using $\mu$ SPECT/CT and fluorescence imaging. Overall, no significant differences between the SPAAC chemistry ligands and their NHS-based counterparts were found ( 2 h p.i., $p>0.05$ ), while ${ }^{111}$ In-labeled ligands outperformed the ${ }^{99 m} \mathrm{Tc}$ ligands.

Conclusion: Here we demonstrate that our newly developed SPAAC-based PSMA ligands show high PSMA-specific tumor targeting. Use of click-chemistry in PSMA ligand development opens up the opportunity for fast, efficient and versatile conjugations of multiple imaging moieties and/or drugs.

\section{Introduction}

Prostate cancer ( $\mathrm{PCa})$ is the second most common cancer in men worldwide, leading to a substantial morbidity and mortality [1]. About $90 \%$ of PCa patients have a localized tumor at initial screening and are candidates for surgery $[2,3]$. The prostate is located between a number of critical structures, and as a consequence the surgeon has to perform very narrow tumor resections $[4,5]$. In approximately $15-65 \%$ of PCa patients, dependent on the disease stage, tumor resection is incomplete caused by positive surgical tumor margins upon removal of the primary tumor and/or incomplete removal of tumor positive lymph nodes in the pelvis [6-8]. Innovative approaches to improve intraoperative tumor visualization could increase the chance of complete surgical resection of all tumor tissue. Here we focused on fluorescence imaging combined with radionuclide detection of PCa using dual-labeled PSMA-targeting ligands [9].

In recent years many PSMA-targeting ligands have been developed, each suitable for different applications (e.g. radionuclide imaging, radionuclide therapy, fluorescent imaging) [10-13]. To develop a versatile platform that enables easy synthesis of PSMA ligands for different purposes, strain-promoted 
azide-alkyne cycloaddition (SPAAC, $\mathrm{N}_{3}$-DBCO) can be used, a well-known form of click chemistry $[14,15]$. Click chemistry reactions proceed with ease under mild nontoxic conditions (i.e. at room temperature in water) tolerating the presence of a wide range of functional groups. Hence, strain-promoted cycloadditions provide fast and highly efficient chemistry to link PSMA ligands to different functional elements $[14,16]$. Nonetheless, there is still uncertainty about the influence of the hydrophobic functional group dibenzocyclooctene that is introduced with SPAAC on the pharmacokinetics and non-specific uptake of the ligands $[14,17,18]$. Therefore, the aim of the current study is to develop small molecule PSMA ligands that can be conjugated to different functional components using SPAAC chemistry $\left(\mathrm{N}_{3}{ }^{-}\right.$ $\mathrm{DBCO}$ ) and to evaluate the effects of the hydrophobic dibenzocyclooctene group on binding affinity, pharmacokinetics and biodistribution of the ligand.

As a first proof-of-concept for the SPAAC chemistry-based PSMA-targeting platform, we synthesized duallabeled PSMA ligands that were conjugated with the near-infrared (NIR) fluorophore IRDye800CW using SPAAC chemistry. The NIR fluorescent dye can be used for fluorescence imaging to allow direct visualization of tumor tissue during surgery. This enables a more precise removal of the primary tumor with less positive surgical margins as a result [19]. Moreover, a chelator for either technetium-99m $\left({ }^{99 \mathrm{~m}} \mathrm{Tc}\right)$ or indium-111 $\left({ }^{111} \mathrm{In}\right)$ labeling was added to the ligand. The addition of a gamma-emitting radionuclide label in the dual-labeled PSMA ligand allows for intraoperative detection of metastatic lymph nodes [20]. Here, we compared the SPAAC-based PSMA-targeting ligands with similar ligands where conventional $N$-hydroxysuccinimide (NHS)-ester coupling of IRDye800CW was used. With this, we could determine the effect of conjugation strategy (SPAAC vs NHS) on the PSMA-binding affinity, biodistribution and pharmacokinetics of these novel dual-labeled ligands. As a secondary aim, we evaluated the differences in affinity and biodistribution of the ${ }^{111}$ In-labeled ligands and their ${ }^{99 \mathrm{~m}} \mathrm{TC}$ labeled equivalents.

\section{Materials And Methods}

\section{Synthesis of ligands}

We synthesized KuE-based PSMA ligands with the chelator mercaptoacetylglycylglycylglycine (MAG3) for ${ }^{99 m}$ Tc labeling, or the chelator 1,4,7,10-tetraazacyclododecane-1,4,7,10-tetraacetic acid (DOTA) for ${ }^{111}$ In labeling using standard Fmoc solid phase chemistry. After cleavage from the resin and intermediate preparative HPLC purification, fluorophore IRDye800CW was conjugated to the ligands via either SPAAC or NHS-ester coupling in solution. Full synthetic procedures can be found in the supplementals (Page 16, Fig. S1-3). The ligands are further referred to as: PSMA-N048 (KuE-linker-DOTA-SPAAC-IRDye800CW), PSMA-N049 (KuE-linker-MAG3-SPAAC-IRDye800CW), PSMA-N050 (KuE-linker-DOTA-NHS-IRDye800CW) and PSMA-N051 (KuE-linker-MAG3-NHS-IRDye800CW) (Fig. 1, Fig. 2A).

\section{Cell culture}


The LS174T colon carcinoma cell line was purchased from the American Type Culture Collection. LS174T-PSMA cells were created by stable transfection with human PSMA using the plasmid pcDNA3.1hPSMA [6]. Wildtype LS174T colon carcinoma cells were used as a control. All cells were cultured in RPMI 1640 medium supplemented with $10 \% \mathrm{FCS}$ and $2 \mathrm{mM}$ glutamine $\left(5 \% \mathrm{CO}_{2}, 37^{\circ} \mathrm{C}\right)$. Additionally, LS174TPSMA cells were cultured in the presence of $0.3 \mathrm{mg} / \mathrm{ml} \mathrm{G} 418$ geneticin.

\section{Radiolabeling}

Indium-111: Ligands (1-20 $\mu \mathrm{g}$,) were radiolabeled under metal-free conditions with ${ }^{111} \mathrm{InCl}_{3}$ (Curium) in $0.5 \mathrm{M}$ 2-(N-morpholino)ethanesulfonic acid (MES) buffer ( $\mathrm{pH}$ 5.5, twice volume of ${ }^{111} \mathrm{InCl}_{3}$ ). Labeling was performed at $90^{\circ} \mathrm{C}$ for 30 minutes [21]. Ethylenediamine-tetraacetic acid (EDTA, $50 \mathrm{mM}$ ) was added to a final concentration of $5 \mathrm{mM}$ after the incubation. Specific activity after labeling ranged from 1-10 MBq/ $\mu \mathrm{g}$. Ligands were purified by a Sep-Pak C18 light cartridge (Waters) and eluted from the cartridge with $50 \%$ ethanol in water.

Technetium-99m: Ligands $\left(1-20 \mu \mathrm{g}\right.$,) were radiolabeled in $45 \mu \mathrm{l}$ ammonium acetate $\left(\mathrm{NH}_{4} \mathrm{Ac}, 0.25 \mathrm{M}, \mathrm{pH} 8\right)$ and $15 \mu \mathrm{l}$ freshly prepared disodium tartate buffer $\left(50 \mathrm{mg} / \mathrm{ml}\right.$ in $\left.0.25 \mathrm{M} \mathrm{NH}_{4} \mathrm{Ac}\right)$, under metal-free conditions. Ascorbic acid buffer was prepared just before labeling ( $3 \mathrm{mg} / \mathrm{ml}$ in $10 \mathrm{mM} \mathrm{HCl})$. Next, $5 \mu \mathrm{l}$ of freshly prepared stannous chloride dihydrate $\left(\mathrm{SnCl}_{2}\right)$ buffer $(4 \mu \mathrm{g} / \mathrm{ml}$ in ascorbic acid buffer) was added

simultaneously with ${ }^{99 \mathrm{~m} \mathrm{TcO}^{-}}{ }_{4}$ in saline, followed by incubation for $30 \mathrm{~min}$ at $90^{\circ} \mathrm{C}$. Specific activity after labeling ranged from 1-30 MBq/ $\mu \mathrm{g}$. Ligands were purified by a Sep-Pak C18 light cartridge (Waters) and eluted from the cartridge with $50 \%$ ethanol in water.

ITLC/HPLC: Radiochemical yield (RCY) was determined by instant thin-layer chromatography (ITLC) using silica gel-coated paper (Agilent Technologies) and $0.1 \mathrm{M}$ ammonium acetate containing $0.1 \mathrm{M}$ EDTA pH $5.5\left({ }^{111} \mathrm{In}\right)$ or $0.1 \mathrm{M}$ Sodium Citrate pH $6.0\left({ }^{99 \mathrm{~m}} \mathrm{TC}\right)$, as the mobile phase. In addition, RCY was measured using reverse-phase high performance liquid chromatography (RP-HPLC) on an Agilent 1200 system (Agilent Technologies) with an in-line radiodetector (Elysia-Raytest). A C18 column (5 $\mu \mathrm{m}, 4.6 \mathrm{x}$ $250 \mathrm{~mm}$; HiChrom) was used at a flow rate of $1 \mathrm{ml} / \mathrm{min}$. We used the following buffer system: buffer $A$, triethylammonium acetate (TEAA, $10 \mathrm{mM}, \mathrm{pH} 7)$; buffer $\mathrm{B}, 100 \%$ methanol; and a gradient of $97-0 \%$ buffer A (35 min).

\section{Binding, internalization and IC50 assay}

Internalization assay: Binding and internalization characteristics of all ligands were compared using LS174T-PSMA and wildtype LS174T cells, cultured to confluency in 6-wells plates. Cells were incubated with 50,000 counts per minute (cpm) ${ }^{111} \mathrm{In}$ - or ${ }^{99 \mathrm{~m}}$ Tc-labeled PSMA ligand (0.1-0.25 pmol/well) in $1 \mathrm{ml}$ binding buffer (RPMI/0.5\% BSA) for $2 \mathrm{~h}$ at $37^{\circ} \mathrm{C}$. Nonspecific binding was determined by co-incubation with 2-(phosphonomethyl)pentane-1,5-dioic acid (2-PMPA, $21.57 \mu \mathrm{M})$. Cells were washed with PBS and incubated with acid buffer $(0.1 \mathrm{M}$ acetic acid, $154 \mathrm{mM} \mathrm{NaCl}, \mathrm{pH} 2.6)$ for 10 minutes at $0^{\circ} \mathrm{C}$ to retrieve the membrane-bound fraction. After this, the membrane-bound fraction was collected, cells were washed, 
lysed with $1.5 \mathrm{ml} 0.1 \mathrm{M} \mathrm{NaOH}$ and cell lysis (intracellular activity) was collected. Membrane-bound activity and intracellular activity fractions were measured in a gamma-counter (2480 WIZARD ${ }^{2}$ Automatic Gamma Counter, PerkinElmer) [6, 22].

$\mathrm{IC}_{50}$ : The $50 \%$ inhibitory concentration $\left(\mathrm{IC}_{50}\right)$ of the ligands was determined using LS174T-PSMA cells in a competitive binding assay. The LS174T-PSMA cells were cultured to confluency in 6-wells plates, followed by incubation on ice for $2 \mathrm{~h}$ in $1 \mathrm{~mL}$ of binding buffer (RPMI/0.5\% BSA) with 50,000 cpm of ${ }^{111}$ In-labeled PSMA-617 and a series of increasing concentrations $(0.01-300 \mathrm{nM})$ of unlabeled PSMA ligands. After incubation, cells were washed with $2 \mathrm{ml} \mathrm{PBS}$ and lysed with $1.5 \mathrm{ml} 0.1 \mathrm{M} \mathrm{NaOH}$. Cell lysis was collected from the plate and the cell-associated activity was measured in a gamma-counter and $\mathrm{IC}_{50}$ values were calculated using GraphPad Prism software version 5.03.

\section{Lipophilicity}

LogD values of all radiolabeled ligands were determined by adding $300,000 \mathrm{cpm}(0.6-1.5 \mathrm{pmol})$ to a mixture of $3 \mathrm{ml}$ PBS (pH 7.4) and $3 \mathrm{ml} \mathrm{n}$-octanol. Tubes were vortexed vigorously for $1 \mathrm{~min}$ and centrifuged for $5 \mathrm{~min}$ at $201 \times \mathrm{g}$. The concentration of radioactivity in a defined volume of each layer was measured in a well-type gamma-counter.

\section{Subcutaneous tumor model}

Animal experiments were performed in 8-10 weeks old male BALB/c nude mice (Janvier). Animals were housed under non-sterile conditions in individually ventilated cages (Blue line IVC, 4-5 mice per cage) with cage enrichment present and free access to water and chlorophyll-free animal chow (Sniff $\mathrm{GmbH}$ ). Mice were subcutaneously inoculated with $3.0 \times 10^{6}$ LS174T-PSMA cells in the right flank and $1.5 \times 10^{6}$ LS174T wildtype cells in the left flank, diluted in $200 \mu \mathrm{L}$ of complete RPMI 1640 medium. When xenografts were approximately $0.1 \mathrm{~cm}^{3}$ (10-14 days after tumor inoculation), tracers were injected intravenously in the tail vain. The biotechnicians performing the s.c. and i.v. injections were blinded for the experimental groups and tumor-bearing mice were block-randomized into groups based on tumor size. All experiments were conducted in accordance to the guidelines of the Revised Dutch Act on Animal Experimentation and approved by the institutional Animal Welfare Committee of the Radboud university medical center.

\section{Biodistribution, fluorescence imaging and $\mu \mathrm{SPECT} / \mathrm{CT}$ imaging}

Mice were injected intravenously with 10 MBq ${ }^{111}$ In-labeled PSMA-N048, -N050 or PSMA-617 as control $(0.3 \mathrm{nmol}$, molar activity $33.3 \mathrm{MBq} / \mathrm{nmol})$ or $15 \mathrm{MBq}{ }^{99 \mathrm{~m}}$ Tc-labeled PSMA-N049 or -N050 (0.3 nmol, molar activity $50 \mathrm{MBq} / \mathrm{nmol}$ ) in PBS/0.5\% BSA. Two hours post injection (p.i.), mice were euthanized by $\mathrm{CO}_{2} / \mathrm{O}_{2}$ asphyxation and images were acquired with the IVIS fluorescence imaging system (Xenogen VivoVision IVIS Lumina II, PerkinElmer), using an acquisition time of $30 \mathrm{~s}$. Subsequently, $\mu$ SPECT/CT images were acquired (U-SPECT II, MILabs) with a $1.0 \mathrm{~mm}$ diameter pinhole mouse collimator tube [23]. Mice were 
scanned for 30 min followed by a CT scan (spatial resolution $160 \mu \mathrm{m}, 65 \mathrm{kV}, 615 \mu \mathrm{A}$ ) for anatomical reference. $\mu S P E C T / C T$ scans were reconstructed with MILabs reconstruction software, using an orderedsubset expectation maximization algorithm, energy windows $154-188 \mathrm{keV}$ and $220-270 \mathrm{keV}$ for ${ }^{111}$ In,

and $126-154 \mathrm{keV}$ for ${ }^{99 \mathrm{~m} T c}, 1$ iteration, 16 subsets, voxel size of $0.4 \mathrm{~mm}$. $\mu$ SPECT/CT scans were analyzed and maximum intensity projections (MIPs) were created using the Inveon Research Workplace software version 4.1 (Siemens Preclinical Solutions). NIRF images were analyzed using Living Image software version 4.2 (Caliper Life Sciences). Tumors, blood, and relevant organs and tissues were dissected, weighed, and radioactivity in each sample was quantified using a well-type gamma-counter. The results were expressed as percentage of injected dose per gram of tissue (\%ID/g).

\section{Pharmacokinetics}

To determine the pharmacokinetics of the ligands, nine groups of five mice received an intravenous injection of $0.3 \mathrm{nmol}{ }^{111} \mathrm{In}$-labeled PSMA-N048 or PSMA-N050 (10 MBq/mouse, molar activity 33.3 $\mathrm{MBq} / \mathrm{nmol}$ ) or ${ }^{99 \mathrm{~m} T \mathrm{Tc}-l a b e l e d}$ PSMA-N049 (5 MBq/mouse, molar activity $16.7 \mathrm{MBq} / \mathrm{nmol}$ ) in PBS/0.5\% BSA. At 2, 4, and $24 \mathrm{~h}$ p.i. mice were euthanized followed by dissection. Tissues of interest were dissected, weighed and measured for radioactivity in a gamma-counter as described above. For each ligand, two mice from the $24 \mathrm{~h}$ groups underwent repeated $\mu$ SPECT /CT and NIRF imaging $(2,4$ and $24 \mathrm{~h}$ p.i.). During imaging, mice were anesthetized with $2.5 \%$ isoflurane inhalation anesthesia and kept warm with a heating pad. Images were acquired and analyzed as described above.

\section{Intraperitoneal tumor model}

LS174T-PSMA cells $\left(1.0 \times 10^{6}\right)$ in $200 \mu \mathrm{l}$ of complete RPMI 1640 medium were injected intraperitoneally and grew for $28 \mathrm{~d}$ after inoculation. Six male BALB/c nude mice with intraperitoneally growing LS174TPSMA tumors were intravenously injected with ${ }^{111}$ In-labeled PSMA-N050 (10 MBq and $0.3 \mathrm{nmol} / \mathrm{mouse}$ ). Two hours p.i., $\mu$ SPECT/CT imaging was performed preoperatively (30 min), followed by NIRF imaging of the mice in supine position after surgical removal of skin, abdominal muscle layers, and peritoneum. After in vivo image acquisition, the visualized tumors were resected, followed by NIRF imaging to control whether residual tumor tissue was in situ.

\section{Statistics}

Statistical analyses were performed with Graphpad Prism, version 5.03. Results are presented as mean \pm standard deviation (SD). Differences in in vitro affinity and tumor/organ uptake in vivo were tested for significance using a one-way ANOVA with a Tukey multiple comparison posttest. Differences were considered significant at $\mathrm{P}<0.05$, two-sided.

\section{Results}

Design and synthesis 
In order to synthesize dual-labeled ligands that fit the active site as well as the entrance funnel of PSMA, we replaced the more $\mathrm{C}$-terminal glutamic acid residue of PSMA-1007 with a lysine [11, 24, 25].

The side chain of this lysine was designed to be oriented towards the exterior of PSMA in the same manner as the side chain of the original glutamic acid is (Fig. S4), providing space for conjugation of multiple functional moieties. To the side chain of this lysine we added another lysine or azidolysine to enable two groups that could be further functionalized with both a metal chelator and a fluorophore. Most of the synthesis was performed on the solid phase, including the addition of a DOTA or MAG3 chelator. After cleavage from the resin we conjugated all ligands in solution with IRDye800CW via either SPAAC $\left(\mathrm{N}_{3}\right.$-DBCO) or NHS-ester based chemistry.

\section{Radiolabeling of the ligands}

RCY of ${ }^{111}$ In-labeled ligands (PSMA-N048 and PSMA-N050) exceeded 93\% in all experiments (specific activity $10 \mathrm{MBq} / \mu \mathrm{g}$ ). RCY of ${ }^{99 \mathrm{~m}} \mathrm{Tc}$ labelled ligands ranged between $15 \%$ and $69 \%$ (specific activity 30 $\mathrm{MBq} / \mu \mathrm{g}$ ). All ligands were purified, resulting in a radiochemical purity $>95 \%$ as determined by ITLC and HPLC. Labelling of all four ligands was stable in human serum at $37^{\circ} \mathrm{C}$ up to 2 hours as determined by RP-HPLC (Fig. S5).

\section{In vitro characterization of the ligands}

To characterize the four ligands we first measured the distribution coefficient ( $\log \mathrm{D}$ at $\mathrm{pH} 7.4$ ), to determine the effect of the dibenzocyclooctene group on the lipophilicity of the PSMA ligand (Fig. 2A). LogD of ${ }^{99 m}$ Tc-PSMA-N049 was -2.4 compared with - 3.5 for its NHS-based equivalent ${ }^{99 m}$ Tc-PSMAN051 ( $p<0.01$ ). The LogD of ${ }^{111}$ In-PSMA-N048 (SPAAC) was -3.2 as compared with -4.4 for ${ }^{111} \mathrm{In}$ PSMA-N50 (NHS) $(p<0.001)$. Hence, as expected the ligands with the dibenzocyclooctene group for SPAAC chemistry were more lipophilic. Use of MAG3 compared with DOTA as a chelator increased lipophilicity as well $(p<0.01)$. In addition to the lipophilicity, we determined the $\mathrm{IC}_{50}$ values of all ligands, which ranged between $184 \mathrm{nM}$ and $475 \mathrm{nM}(p=0.11)$ (Fig. 2A, Fig. S6).

Next, we verified the PSMA-binding potential of our ligands in an in vitro binding and internalization assay using PSMA-positive and negative cells, in which all four ligands showed PSMA-specific binding (Fig. 2B). Use of SPAAC chemistry led to a significantly lower total binding of the ligand; $3.9 \% \pm 0.5 \%$ for 99m Tc-PSMA-N049 and $8.9 \% \pm 0.6 \%$ for ${ }^{111}$ In-PSMA-N048, compared with NHS-conjugated variants; $22.9 \% \pm 1.6 \%$ for ${ }^{99 m}$ Tc-PSMA-N051 and $17.2 \% \pm 3.5 \%$ for ${ }^{111}$ In-PSMA-N050 (Fig. $2 B, p<0.001$ ). As a reference, we determined the total binding of ${ }^{111}$ In-PSMA-617 in our assay, which was $13.4 \% \pm 3.5 \%$. For all ligands, the internalized fraction ranged between $61.0 \%$ and $80.6 \%$ of the total cell-associated activity, and did not differ significantly (Fig. 2A). Minimal binding and internalization were observed in PSMAnegative cells or PSMA-positive cells incubated with an excess of 2-PMPA, demonstrating that, despite their higher lipophilicity, the SPAAC chemistry-based ligands do not suffer from non-specific binding and uptake (Fig. 2B). 
To establish the pharmacokinetics of the SPAAC-based PSMA ligands, the ex vivo biodistribution of ${ }^{99 \mathrm{~m}}$ Tc-PSMA-N049 and ${ }^{111}$ In-PSMA-N048 was determined 2, 4 and $24 \mathrm{~h}$ p.i. in mice bearing LS174T

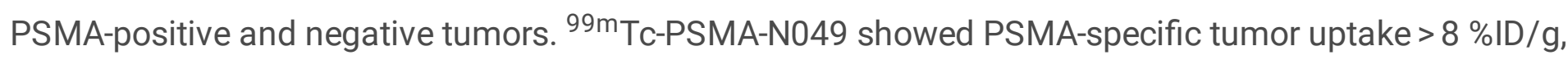
which was stable up to 24 hours after injection (Fig. 3A, Supplementary table 1). Tumor uptake of ${ }^{111} \mathrm{In}$ PSMA-N048 was $23.8 \pm 3.9 \% \mathrm{ID} / \mathrm{g}$ and $22.7 \pm 1.5 \% \mathrm{ID} / \mathrm{g} 2$ and 4 hours p.i., respectively. Yet, after 24 hours p.i. tumor uptake of ${ }^{111} \mathrm{In}$-PSMA-N048 significantly decreased to $17.6 \pm 1.8 \% \mathrm{ID} / \mathrm{g}(\mathrm{p}<0.05)$. Tumor uptake of the ${ }^{111}$ In-labled ligand PSMA-N048 was significantly higher compared with the ${ }^{99 \mathrm{~m}}$ Tc-labeled ligand PSMA-N049 at all three timepoints measured ( $p<0.05$ for all timepoints).

For both ligands, the amount of tracer present in the blood over time decreased from $2-3 \% \mathrm{ID} / \mathrm{g}(2 \mathrm{~h}$ p.i. $)$ to $<0.3 \% \mathrm{ID} / \mathrm{g}$ (24 h p.i.). Furthermore, accumulation of the two ligands in other organs including the PSMA-negative tumor, spleen, liver, prostate and salivary glands was low $(<5 \% \mathrm{ID} / \mathrm{g})$ at the $2 \mathrm{~h}$ p.i. timepoint and further decreased over the course of $24 \mathrm{~h}$, leading to high tumor-to-background ratios at all timepoints measured (Supplementary table 1). $\mathrm{SSPECT/CT}$ and fluorescence imaging of the mice revealed that both ligands clearly visualized the subcutaneous LS174T-PSMA tumors (left flank) up to 4 hours p.i. (Fig. 3B).

\section{Biodistribution comparison of SPAAC-and NHS-based ligands}

To determine the effect of conjugation chemistry (SPAAC vs. NHS) on the in vivo performance of the PSMA ligands, their tumor and normal tissue uptake was determined in mice bearing both a PSMApositive and negative LS174T tumor two hours after injection. Despite the high tumor uptake of all four ligands tested, the NHS-based ligands showed higher tumor accumulation as compared to their SPAACbased counterparts (not significant, $p>0.05$ ). Tumor uptake of PSMA-N048 (SPAAC) and PSMA-N050 (NHS) labeled with ${ }^{111} \mathrm{In}$ were $21.2 \pm 1.2 \% \mathrm{ID} / \mathrm{g}$ and $25.3 \pm 2.0 \% \mathrm{ID} / \mathrm{g}$, respectively. Lowest uptake in the PSMA-expressing tumors was measured for the ${ }^{99 \mathrm{~m}}$ Tc-labeled ligands; $12.0 \pm 1.4 \% \mathrm{ID} / \mathrm{g}$ and $17.7 \pm 3.7$ $\% I D / g$ for PSMA-N049 (SPAAC) and PSMA-N051 (NHS), respectively. In our LS174T s.c. tumor model, uptake of ${ }^{111}$ In-PSMA-N050 was significantly higher than both ${ }^{99 m}$ Tc-labeled ligands $(p<0.01)$ and control ligand ${ }^{111} \mathrm{In}$-PSMA-617 $(18.5 \pm 1.9 \% \mathrm{ID} / \mathrm{g}, \mathrm{p}<0.05)$. All four ligands showed a significantly higher uptake in the PSMA-positive tumor as compared to the PSMA-negative tumor $(p<0.001)$ (Fig. 4A, Supplementary table 2).

All ligands showed rapid blood clearance and minimal uptake in muscle, bone, salivary glands and prostate at 2 hours after injection, leading to high tumor-to-organ ratios (Fig. 4B, Supplementary table 2). The SPAAC chemistry-based ligands showed a higher liver uptake as compared to their NHS-based equivalents; $4.3 \pm 0.3 \% \mathrm{ID} / \mathrm{g}$ (SPAAC) vs. $1.7 \pm 0.3 \% \mathrm{ID} / \mathrm{g}$ (NHS) for ${ }^{99 \mathrm{~m} T c}$ and $3.4 \pm 0.3 \% \mathrm{ID} / \mathrm{g}$ (SPAAC) vs. $1.2 \pm 0.1 \% \mathrm{ID} / \mathrm{g}(\mathrm{NHS})$ for ${ }^{111} \mathrm{In}(\mathrm{p}<0.05)$. Ligand accumulation in the excretory organ, the kidneys, was 
similar for both conjugation strategies, but lower for the ${ }^{99 \mathrm{~m}} \mathrm{Tc}$-labeled ligands (range $73.9-81.6 \% \mathrm{ID} / \mathrm{g}$ ), compared with the ${ }^{111}$ In-labeled ligands $(>120 \%$ ID $/ g, p<0.001)$.

Multimodal imaging of PSMA-positive tumors

To compare the multimodal imaging potential of our SPAAC chemistry-based ligands to that of their NHSbased equivalents, we scanned mice using both a $\mu$ SPECT/CT and a fluorescence scanner. Figure 5 shows representative back- and side-view $\mu$ SPECT/CT images (Fig. 5A) and the corresponding fluorescence images (Fig. 5B) of all four ligands two hours after injection. No large differences in imaging potential were observed between the SPAAC and NHS-based ligands. All ligands could clearly visualize the subcutaneous LS174T-PSMA tumors (right flank), without showing clearly visible uptake in the PSMAnegative LS174T tumors (left flank). In addition, multimodal images visualized high renal tracer accumulation in all mice.

Image-guided surgery with dual-labeled ligands

To evaluate the applicability of the dual-labeled PSMA ligands for image-guided resections in a more clinically relevant setting, we injected six mice with intraperitoneal LS174T PSMA-positive tumors with our ligand ${ }^{111}$ In-PSMA-N050. Two hours after injection of the ligand, a preoperative $\mu$ SPECT/CT scan was acquired, on which multiple i.p. tumors could be visualized (Fig. 6C). Next, we carried out imageguided resection of the i.p. tumors, of which Fig. 6 shows one illustrative example. NIR fluorescence imaging immediately revealed a tumor at the injection site, also visible on $\mu$ SPECT/CT (Fig. 6A1-2, Fig. 6C red arrow). After removal of the tumor at the injection site, fluorescence imaging revealed a large tumor in the abdomen (Fig. 6A3-4) and multiple tumors close to the spleen and pancreas (Fig. 6A3-5), all visible on $\mathrm{SPECT} / \mathrm{CT}$ as well (Fig. $6 \mathrm{C}$ green and pink arrows). Last, small nodules beneath the liver were found (Fig. 6A5, Fig. 6C yellow arrow). All resected tumors showed fluorescent uptake (Fig. 6B). Nevertheless, the kidneys were highly fluorescent as well, which hampered detection of tumor lesions in close proximity to these execratory organs in mice.

\section{Discussion}

In this study we used strain-promoted azide-alkyne cycloaddition (SPAAC) chemistry to synthesize duallabeled PSMA-targeting ligands that could aid in the intraoperative detection and resection of PCa. The SPAAC-chemistry based ligands (PSMA-N048 and PSMA-N049) showed high tumor uptake and fast clearance from other organs. Importantly, performance of the SPAAC-based ligands was similar to their NHS-based equivalents. Together, this suggests the feasibility of SPAAC chemistry as a versatile conjugation strategy in high-affinity PSMA ligand design. Furthermore, the ${ }^{111}$ In-labled ligands showed a significantly higher tumor uptake compared with the ${ }^{99 \mathrm{~m}}$ Tc-labeled counterparts.

Click chemistry has been used in many research fields because of its beneficial characteristics, including high yield, high specificity, and simplicity [26]. More specifically, it enables reasonably fast kinetics under aqueous conditions in the presence of a wide range of functional groups and no high temperatures are required [27]. The recent development of less cytotoxic and copper-free click chemistry reactions such as 
SPAAC has allowed its application in the field of medicine. Hence, the SPAAC-based conjugation strategy presented in this study provides a versatile platform in which PSMA ligands can easily be coupled to different chelators, fluorophores or anti-cancer drugs. For example, it offers the opportunity to click various imaging moieties (e.g. fluorophores, radionuclides or MRI contrast agents) to the ligand for preclinical microscopy, as well as clinical diagnostic, pre- and intraoperative imaging of PCa. Moreover, theranostic tracers could be synthesized that include therapeutic elements such as $\alpha$ - or $\beta$-emitting radionuclides for radioligand therapy, photosensitizers for PSMA-targeted photodynamic therapy or anticancer drugs including chemotherapeutics and immunomodulatory agents. To elucidate the influence of the dibenzocyclooctene group on ligand characteristics, we compared this fast and efficient way of conjugation with conventional NHS-ester chemistry. Withal, the SPAAC-based PSMA ligands showed similar high affinity towards PSMA in vitro, and specific uptake and retention in PSMA-positive tumors in vivo, compared to NHS-based ligands.

Literature has indicated that the dibenzocyclooctene group, present in the ligand after SPAAC chemistry, is rather hydrophobic and could potentially negatively impact the affinity and non-specific binding of small PSMA-targeting ligands in vitro [28, 29]. On the contrary, Wirtz et al. found that higher lipophilicity of their PSMA-I\&T based ligands is beneficial in terms of affinity and internalization, possibly because of ligand interaction with a lipophilic binding pocket of PSMA [30]. LogD determination in our study showed that SPAAC chemistry conjugation of IRDye800CW indeed leads to more lipophilic ligands. We determined the $\mathrm{IC}_{50}$ values of all four ligands to be in the same order of magnitude (184-475 nM), which was lower than that of the high-affinity PSMA-617 ligand $\left(\mathrm{IC}_{50}: 8.5 \mathrm{nM}\right)$. Wirtz et al. found that increasing the lipophilicity of their PSMA-I\&T based ligands is beneficial for ligand internalization in LNCaP cells [30]. In contrast to this, no differences in internalization rate were observed between the SPAAC ligands and their NHS-based counterparts in our study. We, however, observed significantly higher overall binding (membrane bound + internalized fraction) of the NHS-based ligands compared with the SPAAC-variants in LS174T PSMA-positive cells in vitro.

Previously, our group performed a dose and time optimization study with dual-labeled ligands similar to the ones described here and showed that 2 hours p.i. was the optimal time point for imaging [9]. However, higher lipophilicity was found to increase plasma protein binding and alter the pharmacokinetic profile of these PSMA ligands [30,31]. Furthermore, recent literature has shown that other changes in the ligand such as variations in linker and chelator can also cause significant changes in binding affinities, pharmacokinetics and biodistribution [32]. Therefore, we evaluated the pharmacokinetics and optimal imaging time-point of the two more hydrophobic SPAAC-chemistry based ligands (PSMA-N048 and PSMA-N049). Both ligands showed high and specific tumor uptake in PSMA-positive xenografts, with high tumor to background ratios at all timepoints $(2,4$ and $24 \mathrm{~h})$. This resulted in a clear visualization of the tumors using both fluorescence imaging and $\mu \mathrm{SPECT} / \mathrm{CT}$ imaging.

In vivo, higher lipophilicity was reported to increase, bus also decrease tumor uptake of various clickchemistry based tracers $[26,28-30,33]$. In the case of PSMA ligands, higher ligand lipophilicity of PSMAI\&T-based ligands led to an increase in tumor uptake in LNCaP xenografts [30]. In addition, Böhmer et al. 
developed and characterized a copper(I)-catalyzed azide-alkyne cycloaddition (CuAAC) based PSMA ligand for PET imaging called [ $\left.{ }^{18} \mathrm{~F}\right]$ PSMA-MIC01 [34]. This CUAAC-based ligand showed specific tumor uptake in LNCaP xenografts $(11.7 \pm 4.2 \% \mathrm{ID} / \mathrm{g}, 1 \mathrm{~h}$ p.i.) with only minor non-specific uptake in other organs. In this LNCaP tumor model, uptake of the click-chemistry based [ $\left.{ }^{18} \mathrm{~F}\right] \mathrm{PSMA-MIC01}$ was even higher compared with $\left[{ }^{68} \mathrm{Ga}\right] \mathrm{Ga}-\mathrm{PSMA}-11(6.8 \pm 6.3 \% \mathrm{ID} / \mathrm{g})$, conventionally used for PET imaging of PCa patients. In our study, the SPAAC chemistry PSMA ligands showed similar or even higher tumor uptake; 9 $\%$ ID/g for ${ }^{99 m}$ Tc-PSMA-N049 and $21 \%$ ID/g for ${ }^{111}$ In-PSMA-N048 (2 h p.i., LS174T-PSMA xenograft model).

Our ligands, including the SPAAC-based ones, showed low non-specific accumulation in other organs (e.g. PSMA-negative tumor, prostate, salivary glands). Yet, as might be expected, a more than twofold higher liver uptake was measured for the SPAAC variants. These data are in line with the statement of Notni et al. that a lipophilic character of the ligand, induced by large hydrophobic groups (i.e. aromatics such as in dibenzocyclooctene), is prone to increase the fraction of slow hepatobiliary clearance [29]. In addition, the study of Zhang et al. showed that addition of lipophilic quinoline groups in PSMA ligand $\left[{ }^{18} \mathrm{~F}\right] \mathrm{DCFPyl}$ led to a large increase in liver accumulation in mice, primates and PCa patients [33].

For radioguided surgery, ${ }^{99 \mathrm{~m}} \mathrm{Tc}$ and ${ }^{111} \mathrm{In}$ are used because they emit $\mathrm{y}$-photons detectable with a handheld gamma probe. In the present study, as a secondary aim, we evaluated differences between DOTA-based ligands suitable for ${ }^{111}$ In-labeling (PSMA-N048, PSMA-N050) and MAG3-based ligands for labelling with ${ }^{99 \mathrm{~m}} \mathrm{Tc}$ (PSMA-N049, PSMA-N051). Chelation of ${ }^{111} \mathrm{In}$ in DOTA leads to a neutral charge whereas chelation of ${ }^{99 m} \mathrm{Tc}$ in MAG3 leads to a net charge of -1 , which might be advantageous since the introduction of negative charges to increase PSMA affinity and ligand uptake in PSMA-positive tumors was reported in multiple studies [9,35-37]. However, use of MAG3 compared with DOTA as a chelator increased lipophilicity, which could also influence tumor uptake and affinity of the PSMA ligand. Our results show that the ${ }^{111}$ In-DOTA ligands have a significantly higher uptake in the s.c. LS174T PSMApositive tumors compared with the ${ }^{99 \mathrm{~m}} \mathrm{Tc}-\mathrm{MAG} 3$ ligands. Besides favorable in vitro and in vivo properties of the ${ }^{111}$ In-labeled ligands, labeling of ${ }^{99 \mathrm{~m}} \mathrm{Tc}$ in the MAG3 chelator resulted in low RCY (15\%-69\%), which was highly variable between each labeling. In comparison, labeling with ${ }^{111}$ In always resulted in high RCY $(\geq 93 \%$ ). Furthermore, use of MAG3 as a chelator led to more difficulties with the synthesis and overall stability of the PSMA-N051 ligand (data not shown). Consequently, DOTA-based ligands might be preferred over MAG3-based variants.

As shown in the i.p. model presented in this study, tumors located deeper inside the surgical cavity may not be visualized with NIR fluorescence imaging alone due to the absorption of the emitted fluorescent light in overlying tissues $(2-3 \mathrm{~mm})$ [38]. This further emphasizes the importance of dual-labeled NIR fluorescence and radionuclide targeting agents that, in addition to preoperative tumor localization, allow intraoperative mapping of more deeply situated tumor lesions with a gamma probe. The findings of the current study encourage clinical studies with PSMA-targeted dual-labeled ligands to enable image-guided 
complete resection of all PCa lesions during radical prostatectomy, preventing cancer-recurrences and improving the chances for curative PCa surgeries.

In conclusion, we developed four dual-labeled ligands which all showed high PSMA affinity and excellent PSMA-specific tumor uptake. We compared NHS and SPAAC chemistry-based approach to attach the fluorophore IRDye800CW. Overall, no significant differences between the SPAAC chemistry ligands and their NHS-based counterparts were found, while ${ }^{111}$ In-labeled ligands outperformed the ${ }^{99 \mathrm{~m}}$ Tc ligands. These results inspire the use of click chemistry conjugations in PSMA ligand development to enable fast, efficient and easy coupling of various chelators, dyes or even anti-cancer drugs.

\section{Abbreviations}

${ }^{111}$ In: indium-111, $99 \mathrm{~m}$ Tc: technetium-99m, DBCO: Dibenzylcyclooctyne, NHS: N-Hydroxysuccinimide, NIR: Near infrared, PCa: Prostate cancer, PSMA: Prostate specific membrane antigen, SPAAC: strain promoted azide-alkyne cycloaddition.

\section{Declarations}

Acknowledgements: The authors thank Bianca Lemmers-van de Weem, Kitty Lemmens-Hermans and Karin de Haas-Cremers for technical assistance with the animal experiments.

\section{Compliance with ethical standards}

Funding: This work was funded by Else Kröner-Fresenius-Stiftung (2016-A64) and the Dutch Cancer Society (NKB-KWF 10443/2016-1).

Conflict of interest: Y. Derks, D. Löwik, S. Heskamp, P. Laverman and M. Rijpkema are applicants of patent: EP21155853 - PSMA-targeting ligands for multimodal applications.

Ethical approval : All applicable international, national, and/or institutional guidelines for the care and use of animals were followed.

\section{References}

1. Sung H, Ferlay J, Siegel RL, Laversanne M, Soerjomataram I, Jemal A, et al. Global cancer statistics 2020: GLOBOCAN estimates of incidence and mortality worldwide for 36 cancers in 185 countries. CA Cancer J Clin. 2021. doi:10.3322/caac.21660.

2. Wang X, Huang SS, Heston WD, Guo H, Wang BC, Basilion JP. Development of targeted near-infrared imaging agents for prostate cancer. Mol Cancer Ther. 2014;13:2595-606. doi:10.1158/15357163.Mct-14-0422.

3. Wang X, Tsui B, Ramamurthy G, Zhang P, Meyers J, Kenney ME, et al. Theranostic Agents for Photodynamic Therapy of Prostate Cancer by Targeting Prostate-Specific Membrane Antigen. Mol 
Cancer Ther. 2016;15:1834-44. doi:10.1158/1535-7163.Mct-15-0722.

4. Lütje S, Heskamp S, Franssen GM, Frielink C, Kip A, Hekman M, et al. Development and characterization of a theranostic multimodal anti-PSMA targeting agent for imaging, surgical guidance, and targeted photodynamic therapy of PSMA-expressing tumors. Theranostics. 2019;9:2924-38. doi:10.7150/thno.35274.

5. Derks YHW, Löwik DWPM, Sedelaar JPM, Gotthardt M, Boerman OC, Rijpkema M, et al. PSMAtargeting agents for radio- and fluorescence-guided prostate cancer surgery. Theranostics. 2019;9:6824-39. doi:10.7150/thno.36739.

6. Lutje S, Rijpkema M, Franssen GM, Fracasso G, Helfrich W, Eek A, et al. Dual-Modality Image-Guided Surgery of Prostate Cancer with a Radiolabeled Fluorescent Anti-PSMA Monoclonal Antibody. Journal of nuclear medicine: official publication. Society of Nuclear Medicine. 2014;55:995-1001. doi:10.2967/jnumed.114.138180.

7. Yossepowitch O, Briganti A, Eastham JA, Epstein J, Graefen M, Montironi R, et al. Positive surgical margins after radical prostatectomy: a systematic review and contemporary update. European urology. 2014;65:303-13. doi:10.1016/j.eururo.2013.07.039.

8. Eiber M, Fendler WP, Rowe SP, Calais J, Hofman MS, Maurer T, et al. Prostate-Specific Membrane Antigen Ligands for Imaging and Therapy. Journal of nuclear medicine: official publication. Society of Nuclear Medicine. 2017;58:67s-76s. doi:10.2967/jnumed.116.186767.

9. Derks YHW, Rijpkema M, Amatdjais-Groenen HIV, Kip A, Franssen GM, Sedelaar JPM, et al. Photosensitizer-based multimodal PSMA-targeting ligands for intraoperative detection of prostate cancer. Theranostics. 2021;11:1527-41. doi:10.7150/thno.52166.

10. Benešová $M$, Schäfer M, Bauder-Wüst U, Afshar-Oromieh A, Kratochwil C, Mier W, et al. Preclinical Evaluation of a Tailor-Made DOTA-Conjugated PSMA Inhibitor with Optimized Linker Moiety for Imaging and Endoradiotherapy of Prostate Cancer. Journal of nuclear medicine: official publication. Society of Nuclear Medicine. 2015;56:914-20. doi:10.2967/jnumed.114.147413.

11. Cardinale J, Schäfer M, Benešová M, Bauder-Wüst U, Leotta K, Eder M, et al. Preclinical Evaluation of (18)F-PSMA-1007, a New Prostate-Specific Membrane Antigen Ligand for Prostate Cancer Imaging. Journal of nuclear medicine: official publication. Society of Nuclear Medicine. 2017;58:425-31. doi:10.2967/jnumed.116.181768.

12. Weineisen M, Schottelius M, Simecek J, Eiber M, Schwaiger M, Wester H. Development and first in human evaluation of PSMA I\&amp;T - A ligand for diagnostic imaging and endoradiotherapy of prostate cancer. Journal of Nuclear Medicine. 2014;55:1083-.

13. Wüstemann T, Haberkorn U, Babich J, Mier W. Targeting prostate cancer: Prostate-specific membrane antigen based diagnosis and therapy. Med Res Rev. 2019;39:40-69. doi:10.1002/med.21508.

14. Mushtaq S, Yun SJ, Jeon J. Recent Advances in Bioorthogonal Click Chemistry for Efficient Synthesis of Radiotracers and Radiopharmaceuticals. Molecules. 2019;24. doi:10.3390/molecules24193567. 
15. Nwe K, Brechbiel MW. Growing applications of "click chemistry" for bioconjugation in contemporary biomedical research. Cancer Biother Radiopharm. 2009;24:289-302. doi:10.1089/cbr.2008.0626.

16. Rossin R, Robillard MS. Pretargeted imaging using bioorthogonal chemistry in mice. Curr Opin Chem Biol. 2014;21:161-9. doi:10.1016/j.cbpa.2014.07.023.

17. Kim E, Koo H. Biomedical applications of copper-free click chemistry: in vitro, in vivo, and ex vivo. Chem Sci. 2019;10:7835-51. doi:10.1039/c9sc03368h.

18. Hensbergen AW, van Willigen DM, Welling MM, van der Wijk FA, de Korne CM, van Oosterom MN, et al. Click Chemistry in the Design and Production of Hybrid Tracers. ACS Omega. 2019;4:12438-48. doi:10.1021/acsomega.9b01484.

19. Lütje S, Rijpkema M, Helfrich W, Oyen WJ, Boerman OC. Targeted radionuclide and fluorescence dualmodality imaging of cancer: preclinical advances and clinical translation. Mol Imaging Biol. 2014;16:747-55. doi:10.1007/s11307-014-0747-y.

20. Rauscher I, Eiber M, Jilg CA, Gschwend JE, Maurer T. [PSMA-radioguided surgery in localized recurrent prostate cancer: Current and future aspects]. Urologe A. 2017;56:18-23. doi:10.1007/s00120-016-0275-2.

21. Brom M, Joosten L, Oyen WJ, Gotthardt M, Boerman OC. Improved labelling of DTPA- and DOTAconjugated peptides and antibodies with 111In in HEPES and MES buffer. EJNMMI Res. 2012;2:4. doi:10.1186/2191-219x-2-4.

22. Lutje S, van Rij CM, Franssen GM, Fracasso G, Helfrich W, Eek A, et al. Targeting human prostate cancer with $111 \mathrm{ln}$-labeled D2B IgG, $F\left(a b^{\prime}\right) 2$ and Fab fragments in nude mice with PSMA-expressing xenografts. Contrast media molecular imaging. 2015;10:28-36. doi:10.1002/cmmi.1596.

23. van der Have F, Vastenhouw B, Ramakers RM, Branderhorst W, Krah JO, Ji C, et al. U-SPECT-II: An Ultra-High-Resolution Device for Molecular Small-Animal Imaging. Journal of nuclear medicine: official publication. Society of Nuclear Medicine. 2009;50:599-605. doi:10.2967/jnumed.108.056606.

24. Barinka $C$, Byun $Y$, Dusich $C L$, Banerjee SR, Chen Y, Castanares $M$, et al. Interactions between human glutamate carboxypeptidase II and urea-based inhibitors: structural characterization. J Med Chem. 2008;51:7737-43. doi:10.1021/jm800765e.

25. Barinka CN, Motlova Z. L. X-ray structure of human glutamate carboxypeptidase II (GCPII) in complex with a urea based inhibitor PSMA 1007. Protein Data Bank 2018;PDB ID: 505T.

26. Takayama Y, Kusamori K, Nishikawa M. Click Chemistry as a Tool for Cell Engineering and Drug Delivery. Molecules. 2019;24. doi:10.3390/molecules24010172.

27. Kelly J, Amor-Coarasa A, Nikolopoulou A, Kim D, Williams C Jr, Ponnala S, et al. Synthesis and preclinical evaluation of a new class of high-affinity (18)F-labeled PSMA ligands for detection of prostate cancer by PET imaging. Eur J Nucl Med Mol Imaging. 2017;44:647-61. doi:10.1007/s00259-016-3556-5.

28. Okoye NC, Baumeister JE, Khosroshahi FN, Hennkens HM, Jurisson SS. Chelators and metal complex stability for radiopharmaceutical applications. Radiochim Acta. 2019;107:1087-120. 
doi:10.1515/ract-2018-3090.

29. Notni J, Wester HJ. A Practical Guide on the Synthesis of Metal Chelates for Molecular Imaging and Therapy by Means of Click Chemistry. Chemistry. 2016;22:11500-8. doi:10.1002/chem.201600928.

30. Wirtz M, Schmidt A, Schottelius M, Robu S, Günther T, Schwaiger M, et al. Synthesis and in vitro and in vivo evaluation of urea-based PSMA inhibitors with increased lipophilicity. EJNMMI Res. 2018;8:84. doi:10.1186/s13550-018-0440-2.

31. Deberle LM, Tschan VJ, Borgna F, Sozzi-Guo F, Bernhardt P, Schibli R, et al. Albumin-Binding PSMA Radioligands: Impact of Minimal Structural Changes on the Tissue Distribution Profile. Molecules. 2020;25. doi:10.3390/molecules25112542.

32. Bao K, Lee JH, Kang H, Park GK, El Fakhri G, Choi HS. PSMA-targeted contrast agents for intraoperative imaging of prostate cancer. Chem Commun (Camb). 2017;53:1611-4. doi:10.1039/c6cc09781b.

33. Zhang X, Wu Y, Zeng Q, Xie T, Yao S, Zhang J, et al. Synthesis, Preclinical Evaluation, and First-inHuman PET Study of Quinoline-Containing PSMA Tracers with Decreased Renal Excretion. J Med Chem. 2021;64:4179-95. doi:10.1021/acs.jmedchem.1c00117.

34. Böhmer VI, Szymanski W, van den Berg KO, Mulder C, Kobauri P, Helbert H, et al. Modular Medical Imaging Agents Based on Azide-Alkyne Huisgen Cycloadditions: Synthesis and Pre-Clinical Evaluation of (18) F-Labeled PSMA-Tracers for Prostate Cancer Imaging. Chemistry. 2020;26:1087181. doi:10.1002/chem.202001795.

35. Huang SS, Wang X, Zhang Y, Doke A, DiFilippo FP, Heston WD. Improving the biodistribution of PSMA-targeting tracers with a highly negatively charged linker. Prostate. 2014;74:702-13. doi:10.1002/pros.22789.

36. Banerjee SR, Foss CA, Castanares M, Mease RC, Byun Y, Fox JJ, et al. Synthesis and evaluation of technetium-99m- and rhenium-labeled inhibitors of the prostate-specific membrane antigen (PSMA). J Med Chem. 2008;51:4504-17. doi:10.1021/jm800111u.

37. Uspenskaya AA, Nimenko EA, Machulkin AE, Beloglazkina EK, Majouga AG. The importance of linkers in the structure of PSMA ligands. Curr Med Chem. 2021. doi:10.2174/0929867328666210804092200.

38. Kovar JL, Simpson MA, Schutz-Geschwender A, Olive DM. A systematic approach to the development of fluorescent contrast agents for optical imaging of mouse cancer models. Anal Biochem. 2007;367:1-12. doi:10.1016/j.ab.2007.04.011.

\section{Figures}



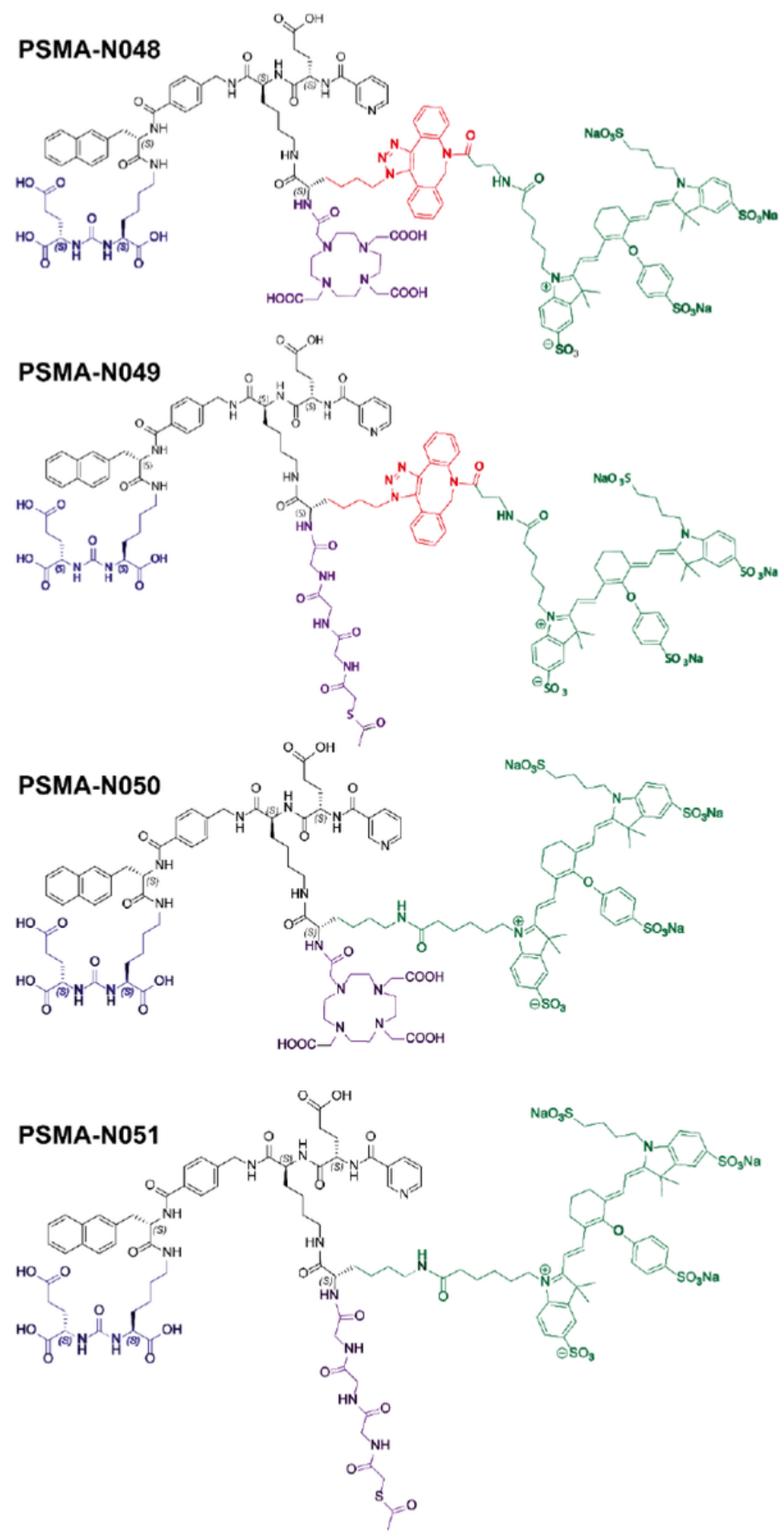

\section{Figure 1}

Chemical structures of PSMA-N048, PSMA-N049, PSMA-N050 and PSMA-N051. Ligands consisting of KuE (blue), linker (black), SPAAC (red), IRDye800CW (green) and MAG3 or DOTA chelator (purple). 
A

\begin{tabular}{|c|c|c|c|c|}
\hline Name & Structure & IC50 (nM) & LogD & $\begin{array}{c}\text { Internalization } \\
\text { ratio (\%) }\end{array}$ \\
\hline \hline PSMA-N048 & DOTA-SPAAC-IRDye800CW & $184.4(95 \% \mathrm{Cl}: 91.0-373.6)$ & $-3.2 \pm 0.16$ & $80.6 \pm 11.6$ \\
\hline PSMA-N049 & MAG3-SPAAC-IRDye800CW & $358.7(95 \% \mathrm{Cl}: 226.4-568.4)$ & $-2.4 \pm 0.39$ & $71.9 \pm 13.7$ \\
\hline PSMA-N050 & DOTA-NHS-IRDye800CW & $312.3(95 \% \mathrm{Cl}: 180.8-539.3)$ & $-4.4 \pm 0.01$ & $74.1 \pm 20.3$ \\
\hline PSMA-N051 & MAG3-NHS-IRDye800CW & $474.9(95 \% \mathrm{Cl}: 271.6-830.5)$ & $-3.5 \pm 0.01$ & $61.0 \pm 12.8$ \\
\hline PSMA-617 & & $8.5(95 \% \mathrm{Cl}: 5.1-14.4)$ & $-4.1 \pm 0.01$ & $48.4 \pm 12.4$ \\
\hline
\end{tabular}

B

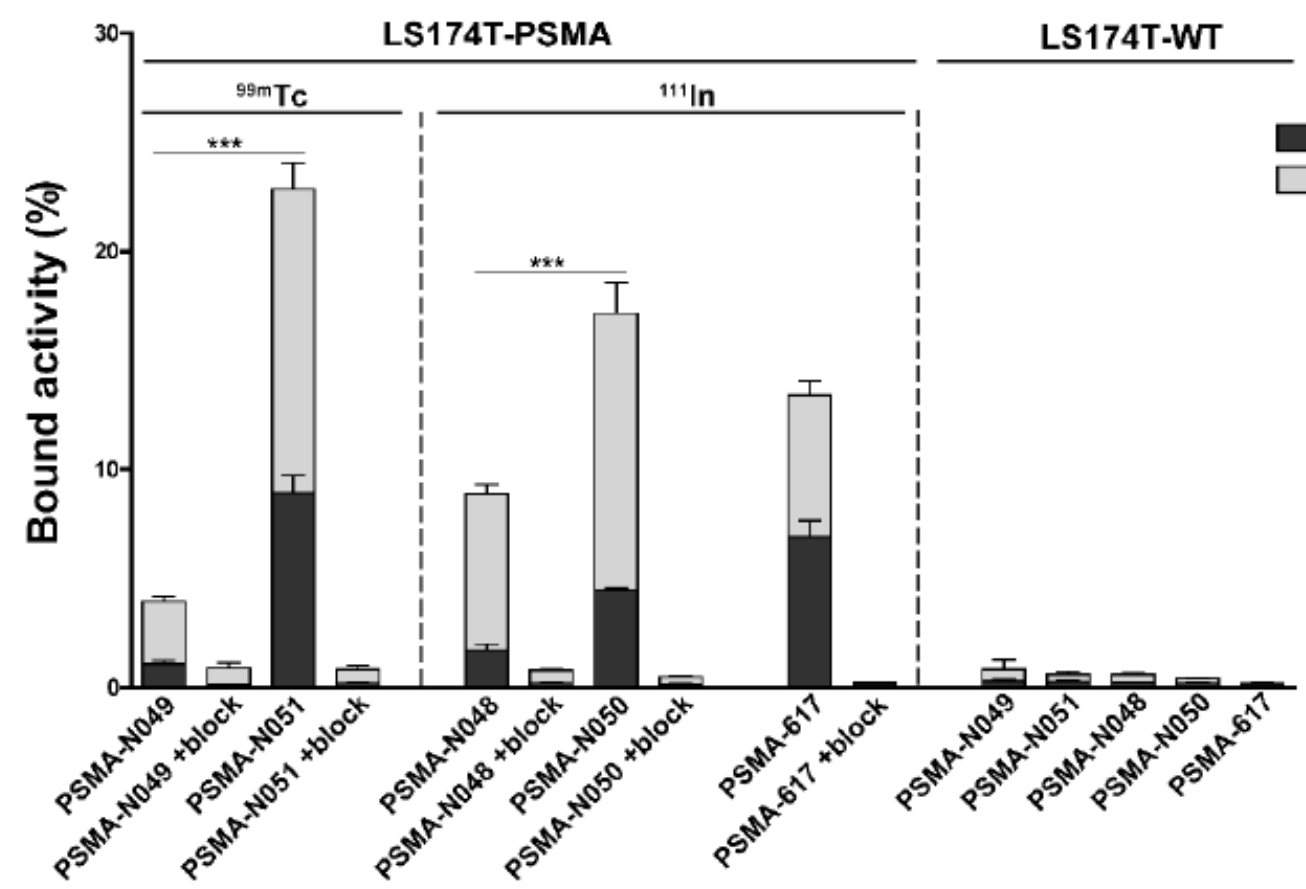

Receptor bound fraction Internalized fraction

Figure 2

In vitro characterization of PSMA-N048, PSMA-N049, PSMA-N050 and PSMA-N051. (A) IC50 values of ligands as determined in competitive binding assays using LS174T-PSMA cells. Lipophilicity of ligands expressed in logD values. Internalization ratio as determined in LS174T-PSMA cells (B) Membrane binding and internalization kinetics of PSMA-N048, -N049, -N050 and -N051 in LS174T PSMA-positive and negative cells. Non-specific binding was determined by blocking with an excess of 2-PMPA $(50 \mu \mathrm{g})$. PSMA-617 was added as a positive control. 

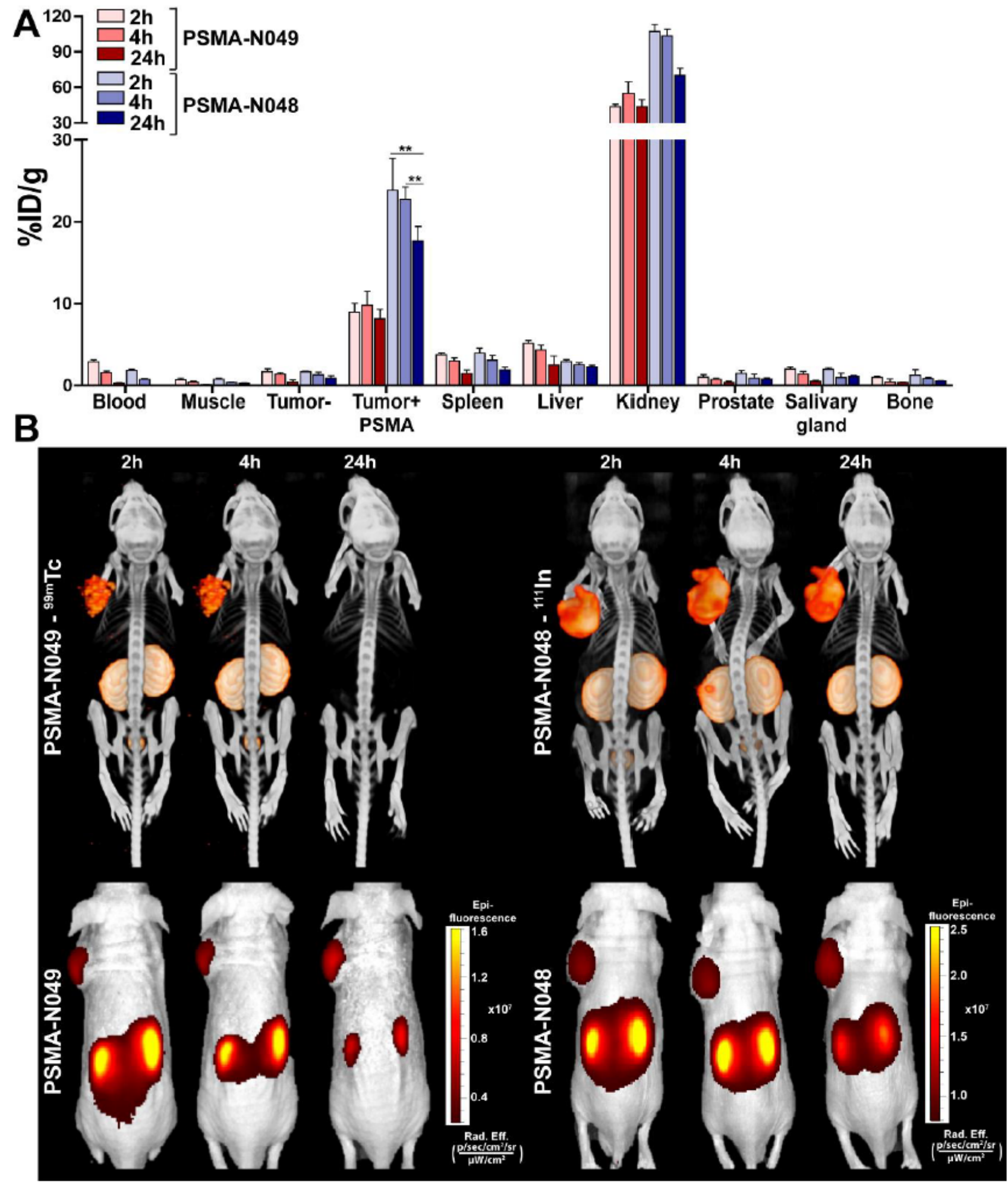

Figure 3

In vivo pharmacokinetics of 99mTc-PSMA-N049 and 111In-PSMA-N048. (A) Biodistribution as determined after dissection of 99mTc-PSMA-N049 (3 MBq/mouse) and 1111n-PSMA-N048 (10 $\mathrm{MBq} /$ mouse) 2, 4 and 24 hours p.i. (0.3 nmol, $\mathrm{n}=5$ /group). Biodistribution was determined in mice bearing subcutaneous LS174T-PSMA and LS174T wildtype xenografts. Data is expressed as \%ID/g \pm SD, ** indicates $p<0.01$. (B) Representative $\mu$ SPECT/CT scans and fluorescence images of mice with s.c. 

MBq/mouse) and 111In-PSMA-N048 (10 MBq/mouse) 2, 4 and 24 hours p.i.
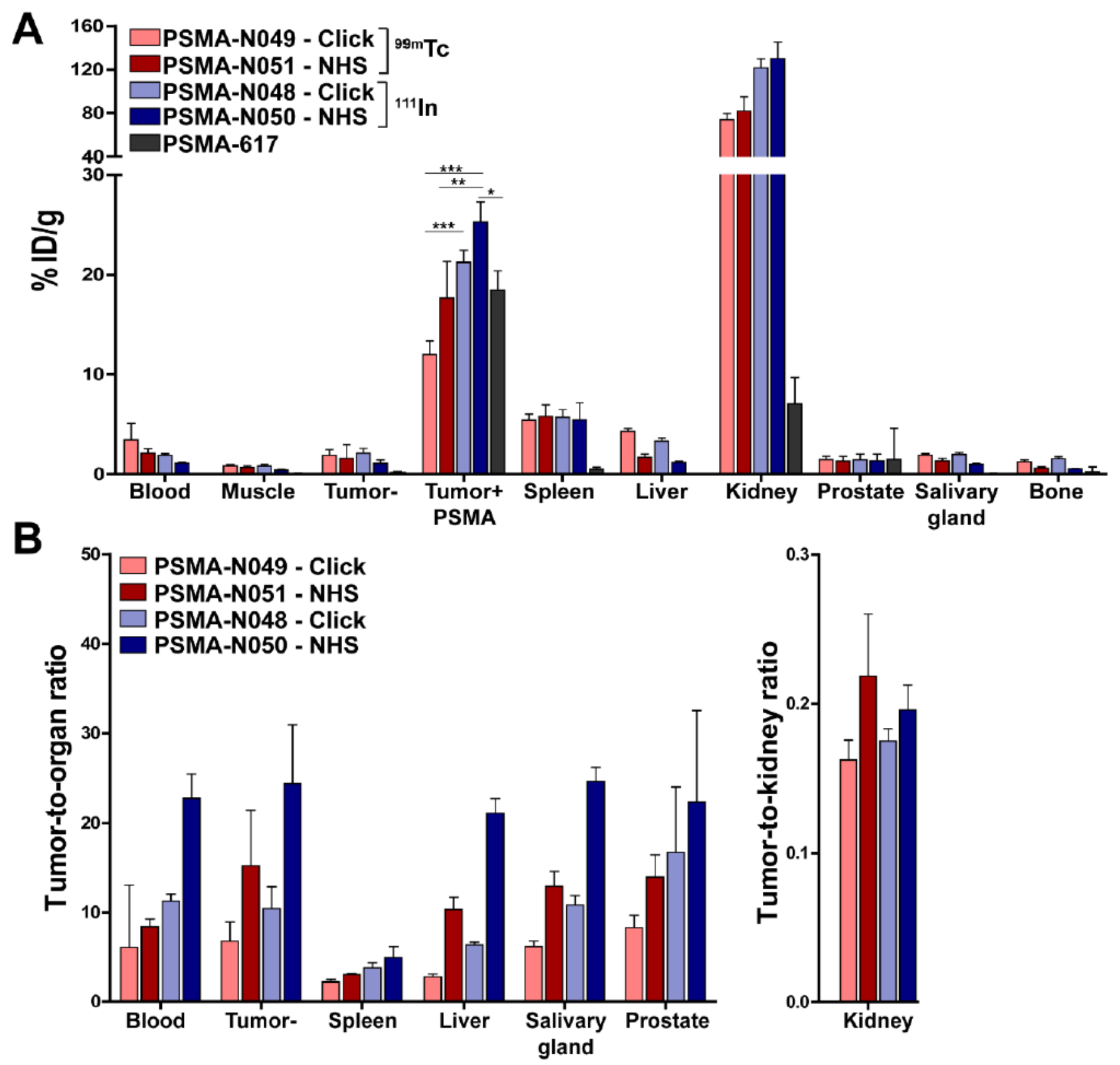

Figure 4

In vivo comparison of PSMA-N048, PSMA-N049, PSMA-N050 and PSMA-N051. (A) Biodistribution as determined after dissection and $(B)$ resulting tumor-to-organ ratios of four $111 \mathrm{ln}$ - (10 MBq/mouse) or 99mTc-labeled (15 MBq/mouse) ligands and positive control PSMA-617 ( $0.3 \mathrm{nmol}, 2 \mathrm{~h}$ p.i., $\mathrm{n}=5$ /group). Biodistribution was determined in mice bearing subcutaneous LS174T-PSMA and LS174T wildtype xenografts. Data is expressed as \%ID/g $\pm S D$, * indicates $p<0.05$, ** indicates $p<0.01$, *** indicates $p<$ 0.001 . 


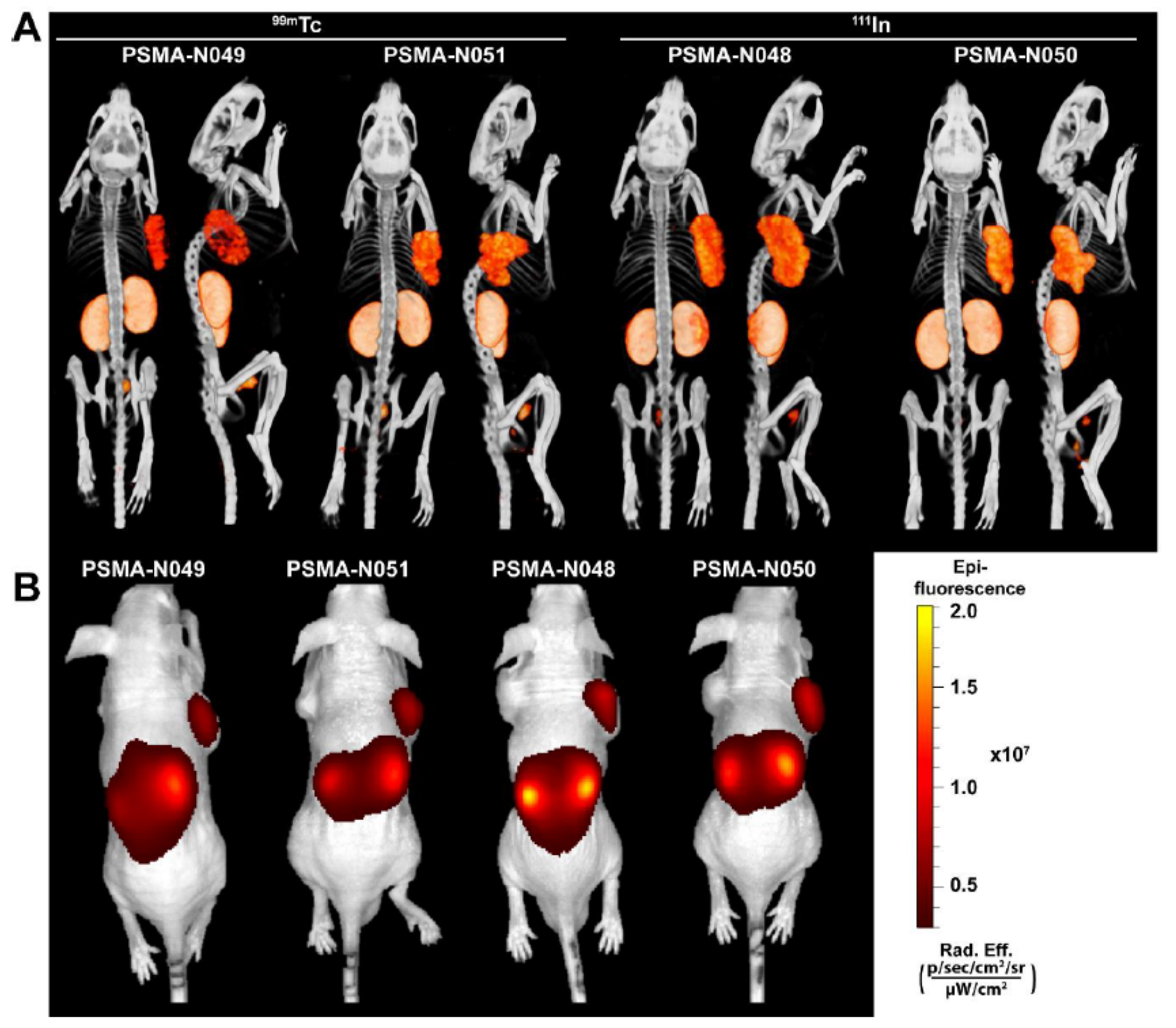

Figure 5

All ligands clearly visualize PSMA-positive tumors using both $\mu$ SPECT/CT and fluorescence imaging. Representative $\mu$ SPECT/CT scans (A) and fluorescence images (B) of mice with s.c. LS174T-PSMA (right) and wildtype LS174T (left) tumors after i.v. injection of $111 \mathrm{ln}$ - (10 MBq/mouse) or 99mTc-labeled (15 $\mathrm{MBq} /$ mouse) ligands (0.3 nmol, 2 h p.i.). 


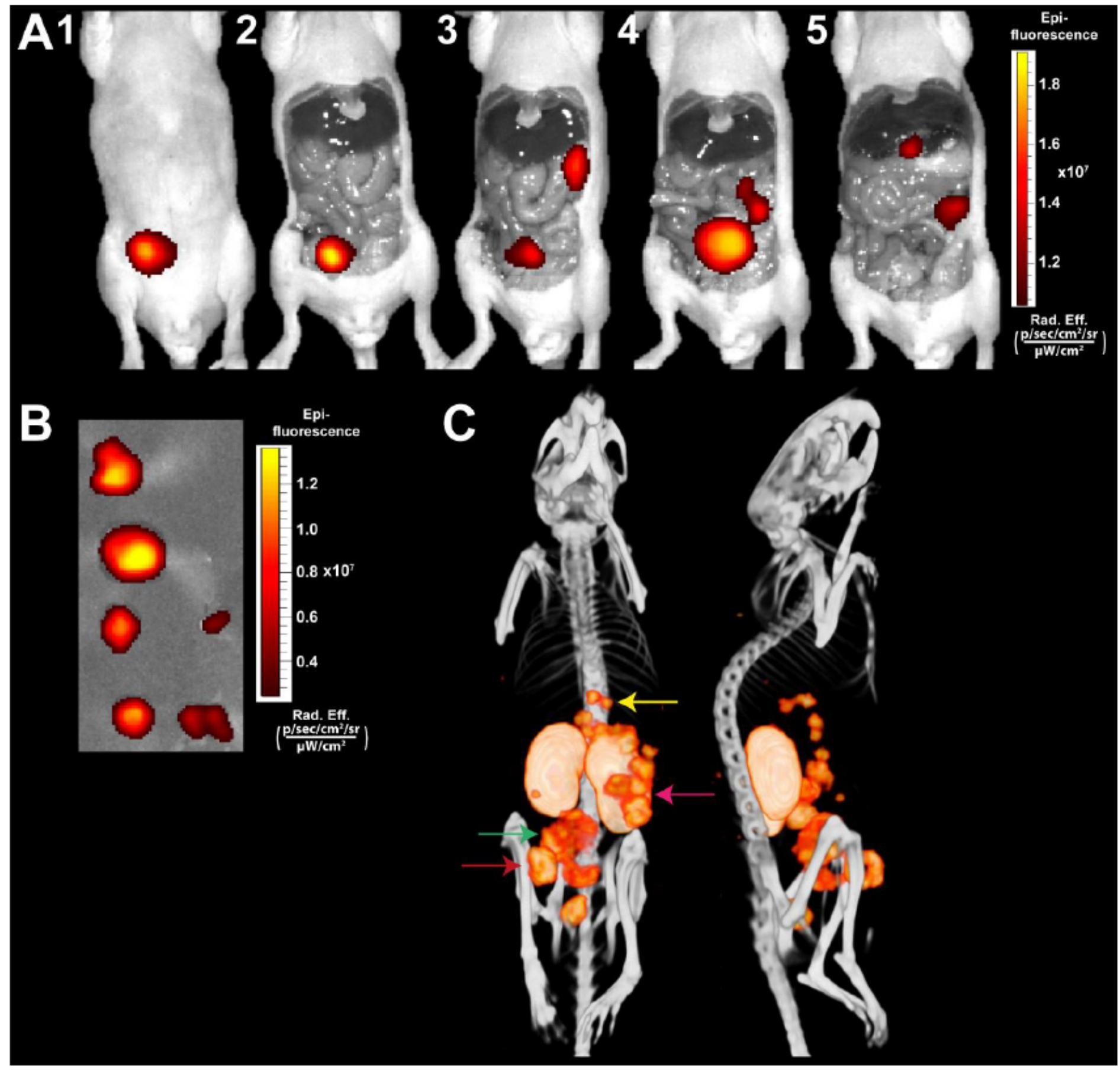

\section{Figure 6}

Multimodal fluorescence and $\mu$ SPECT/CT imaging of intraperitoneal PSMA-positive tumors using $111 \mathrm{ln}$ PSMA-N050. Mouse with several intraperitoneal LS174T-PSMA tumors located at different depths in the peritoneal cavity. (A) Same scale NIRF images of mouse with several intraperitoneal tumors after i.v. injection of 111 In-labeled PSMA-N050 (0.3 nmol, $10 \mathrm{MBq} / \mathrm{mouse}, 2 \mathrm{~h}$ p.i.). (B) NIRF image of removed tumors (C) Corresponding $\mu$ SPECT/CT images in supine and left lateral positions.

\section{Supplementary Files}


This is a list of supplementary files associated with this preprint. Click to download.

- 20210920SupplementalsSPAACbasedPSMAtargetingligands.pdf 